\title{
Characterisation of physico-mechanical properties and degradation potential of calcium alginate beads for use in embolisation
}

\author{
Richard E. J. Forster · Frank Thürmer • Christine Wallrapp • \\ Andrew W. Lloyd · Wendy Macfarlane · Gary J. Phillips • \\ Jean-Pierre Boutrand • Andrew L. Lewis
}

Received: 1 March 2010/Accepted: 1 April 2010/Published online: 22 April 2010

(C) The Author(s) 2010. This article is published with open access at Springerlink.com

\begin{abstract}
High molecular weight alginate beads with $59 \%$ mannuronic acid content or $68 \%$ guluronic acid were prepared using a droplet generator and crosslinked in calcium chloride. The alginate beads were compared to current embolisation microspheres for compressibility and monitored over 12 weeks for size and weight change at $37^{\circ} \mathrm{C}$ in low volumes of ringers solutions. A sheep uterine model was used to analyse bead degradation and inflammatory response over 12 weeks. Both the in vitro and in vivo data show good delivery, with a compressibility similar to current embolic beads. In vitro, swelling was noted almost immediately and after 12 weeks the first signs of degradation were noted. No difference was noted in vivo. This study has shown that high molecular weight alginate gel beads were well tolerated by the body, but beads associated with induced thrombi were susceptible to inflammatory cell infiltration. The beads were shown to be easy to handle and were still observable after 3 months in vivo. The beads were robust enough to be delivered
\end{abstract}

R. E. J. Forster · A. L. Lewis ( $\square)$

Biocompatibles UK Ltd., Farnham Business Park, Weydon

Lane, Farnham, Surrey GU9 8QL, UK

e-mail: andrew.lewis@biocompatibles.com

F. Thürmer · C. Wallrapp

CellMed AG, Industriestraße 19, 63755 Alzenau, Germany

R. E. J. Forster · A. W. Lloyd · W. Macfarlane · G. J. Phillips Biomedical Materials Research Group, School of Pharmacy and Biomolecular Sciences, University of Brighton, Mouslecoomb, Brighton, E.Sussex BN2 4GJ, UK

J.-P. Boutrand

Biomatech S.A., Zone Industrielle de I'Islon, 115 Rue Pasteur, 38670 Chasse-sur-Rhone, France through a 2.7 Fr microcatheter. This study has demonstrated that high molecular weight, high purity alginate bead can be considered as semi-permanent embolisation beads, with the potential to bioresorb over time.

\section{Introduction}

Polysaccharides, such as alginates, are widely used in food, cosmetic and medical fields due to their structural properties, biocompatibility and safety. When alginate is combined with divalent cations a hydrogel is formed that exhibits a level of biocompatibility that can encapsulate cells [1]. Previous studies have shown that purity can affect the biocompatibility of alginate implants [2]. Alginate is a copolymer composed of mannuronate (M) and guluronate (G) stereomonomers [3, 4]. The affinity of a divalent cation (i.e. calcium) is stronger for $\mathrm{G}$ isomers due to the steric arrangement of the atoms $[4,5]$. Two G monomers form an "egg-box" in which a calcium ion sits and crosslinks two polymer chains [4]. Consequently the M:G ratio will effect the crosslinking capacity and thus the physical properties of a calcium alginate gel [6].

Alginate is also a biodegradable polymer; the backbone can be degraded via hydrolysis of the glycosidic bonds [7] or an alginate gel can dissolve due to the elution of the cross-linking calcium cation [6]. Molecular weight has been shown to affect alginate polymer chain degradation [8] and the elution of the crosslinking calcium can also lead to dissolution of gel [9]. This paper considers both high $\mathrm{M}$ and high $G$ calcium crosslinked alginate gel beads as devices for use in embolisation.

Therapeutic embolisation is the intentional introduction of a (foreign) embolic material into a blood vessel in order to reduce or completely obstruct the blood flow. This 
procedure is commonly used to treat hypervascularised tumours and arteriovenous malformations (AVMs). The choice of embolic agent depends on the desired level of occlusion down the vascular tree and permanency. The ideal occlusion technique is one that allows for accurate guidance and delivery to the target with low risk of injury to normal structures. To achieve this, interventional radiologists (IRs) are of the opinion that the embolic agent requires a combination of a number of attributes [10]. In handling the agent, it must be either radiopaque or have the ability to be mixed into a radiopaque suspension to enable visualisation during delivery. Simplicity and reliability of delivery is preferred (glue is notoriously difficult to handle for instance), without blocking of the catheters used to deliver the agents. Adaptability to allow selective and rapid occlusion of various blood vessels sizes and types is desirable; and an ability to reach distal vascular beds is particularly important in the treatment of tumours. If possible, the agent should have amenability to trouble shooting/salvage in case of complications or device malfunction. Other important considerations include biocompatibility and cost competitiveness.

One important and growing area of embolotherapy is uterine artery embolisation (UAE), or sometimes referred to as uterine fibroid embolisation (UFE). Since its fist report in 1995 [11] it is estimated that more that 100,000 UAE procedures have been performed (mainly in the USA and Europe) [12]. Outcomes from a large US registry [13] and four separate (albeit relatively small) randomised trials over the last few years [14-17] demonstrate that UAE is safe and effective, gives similar results to hysterectomy but with faster and shorter recovery and uterine preservation. This is balanced against the need to retreat some patients following UAE. Given that some patients opt for UAE as they wish to maintain the potential for future pregnancy, there is some debate over whether the embolisation agent should be permanent or temporary in nature. Gelatin sponge (or Gelfoam $^{\circledR}$ ) provides the only current degradable option, which is non-calibrated, has an irreproducible degradation rate and can induce a strong inflammatory response. A calibrated microspherical embolisation agent with controlled degradability could therefore fulfil a role in the clinical practice of UAE.

This paper reports the results from some in vitro and in vivo models used to assess all the key necessary attributes of alginate beads as an embolisation device. The in vitro tests examined bead compressibility compared to other embolic beads on the market, microcatheter deliverability and bead degradation. A sheep uterine artery embolisation model, designed to mimic UAE conditions, was used to assess bead deliverability, biocompatibility and degradation over 12 weeks in vivo.

\section{Experimental}

\subsection{Materials}

Dry kelp is used for the production of Phycomer (CellMed AG, Alzenau, Germany), a highly purified sodium alginate with a markedly low impurity profile, made by a proprietary patented purification process [18]. Phycomer E01, a high $\mathrm{M}$ alginate with a mannuronic acid content about $59 \%$, is isolated from the brown algae species Laminaria pallida. It has a molecular weight of greater than $800,000 \mathrm{~g} / \mathrm{mol}$ with a typical viscosity of a $0.2 \% \mathrm{w} / \mathrm{v}$ solution being 10-60 mPa.s. Phycomer E01 has properties ideally suited for the production of soft, biodegradable nonpermanent implants.

Phycomer C01, which is isolated from Laminaria hyperborea, is a high $\mathrm{G}$ alginate with a guluronic acid content about $68 \%$ and a molecular weight of around 400,000 g/ mol, producing a $0.2 \% \mathrm{w} / \mathrm{v}$ solution viscosity of 513 mPa.s. Phycomer C01 forms stiffer gels with tighter pores than Phycomer E01 and is used for the immunoisolation of cellular implants, such as GLP-1 producing CellBeads which are under clinical evaluation for the treatment of haemorrhagic stroke [19] and pre-clinical evaluation for the treatment of acute myocardial infarction [20].

The beads used in the compressibility comparison tests were 900-1200 $\mu \mathrm{m}$ Bead Block ${ }^{\mathrm{TM}}$; (Biocompatibles UK Ltd, UK), 900-1200 $\mu \mathrm{m}$ DC Bead ${ }^{\mathrm{TM}}$; (Biocompatibles UK Ltd, UK), 900-1200 $\mu \mathrm{m}$ Embosphere ${ }^{\circledR}$ (Biosphere Medical Inc., USA) and Countour $\mathrm{SE}^{\mathrm{TM}}$ (Boston Scientific Inc. USA).

\subsection{Methods}

\subsubsection{Bead preparation}

The beads were prepared by dissolving high molecular weight sodium alginate at a concentration of $0.6 \%(\mathrm{w} / \mathrm{v})$ in saline. Using a droplet generator similar to that described elsewhere [21], a stream of the alginate solution was injected through a needle bore using a motorized syringe and cut using a jet of hydrated compressed air, forming small alginate solution droplets. The solution droplets were crosslinked in a $100 \mathrm{mM}$ calcium chloride solution placed underneath the syringe. Bead diameter could be controlled by varying key parameters such as the solution injection rate, airflow and needle size. The mean diameters $( \pm S D)$ of the beads used in most experiments were $506 \mu \mathrm{m}$ $( \pm 18 \mu \mathrm{m})$ and $506 \mu \mathrm{m}( \pm 21 \mu \mathrm{m})$ in Ringers solution for high $\mathrm{G}$ and high $\mathrm{M}$ beads respectively. Larger beads made using the same procedure were generated for compression testing. All beads were produced under aseptic conditions 
to maintain sterility, as conventional sterilisation methods such as irradiation cause degradation of the alginate and affect its ability to gel [22].

\subsubsection{Compression testing}

The required force necessary to compress the alginate beads was assessed using a flat $5 \mathrm{~mm}$ cylindrical probe attached to an Instron Materials Testing Systems Model 4411 (Instron High Wycombe, UK) mounted with a $50 \mathrm{~N}$ load cell. A close packed monolayer of beads (900$1100 \mu \mathrm{m})$ in Ringers solution was placed on a flat surface underneath the probe. Excess Ringers solution was wicked from the sample using a tissue. The probe was positioned $1.2 \mathrm{~mm}$ above the plate. The probe was then extended by $0.85 \mathrm{~mm}$ at a speed of $5 \mathrm{~mm} / \mathrm{min}$ and the load data was recorded. The modulus was calculated from the initial compression gradient of a stress/strain plot of the compression force. Since the resistivity of a hydrogel to compression is sensitive to conditions such as humidity and temperature all beads were tested within $2 \mathrm{~h}$ of each other to minimise these effects. Commercially available embolic microspheres were tested for comparison. The embolic beads tested were High $\mathrm{G}$ alginate, High $\mathrm{M}$ alginate, DC Bead, BeadBlock, Contour SE and Embosphere.

\subsubsection{Deliverability (in vitro)}

In order to assess simplicity of delivery technique and reliability of the delivery mechanism, each of the bead formulations were delivered down a Progreat 2.7 Fr catheter (Teumo, Japan) three times. Alginate gel beads were suspended at a 1/10 dilution in a 50:50 mix of saline (0.9\%) and contrast medium (Omnipaque, GE Healthcare, USA). Each solution was placed on a roller mixer for 3-5 min. Half of the sample volume was drawn into a reservoir luer lock syringe; this was connected to a three way connector and thoroughly mixed with an attached $3 \mathrm{ml}$ delivery syringe. Post mixing, the product was drawn and delivered from the $3 \mathrm{ml}$ syringe. Once delivered a further two $3 \mathrm{ml}$ aliquots were drawn, mixed and delivered.

The delivery was scored using a $0-5$ scoring system $(0$ indicating a saline like delivery and 5 indicating a complete blockage). The catheter was then flushed with saline and the process was repeated with the second half of the bead/ solution mix. Post delivery the beads were assessed for bead fragmentation using an Olympus phase contrast microscope.

\subsubsection{Degradation (in vitro)}

Alginate gel bead degradation analysis was monitored in vitro by measuring the elution of calcium from the beads using ion chromatography (IC) and size/weight change of the hydrated beads using an Olympus phase contrast microscope. To mimic the minimal fluid embolic bead would be exposed to in an embolic environment in vivo, only $1 \mathrm{ml}$ Ringers solution was placed in a centrifuge tube with $0.5 \mathrm{ml}$ alginate beads measured into centrifuge inserts with $5 \mu \mathrm{m}$ pores (Ultrafree-MC PVDF durapore; Millipore, UK). The samples were placed in an oven at $37^{\circ} \mathrm{C}$ until a testing time point, when the samples were centrifuged (JOUAN CR312, Paris, France) at 300G for $5 \mathrm{~min}$. The solution collected after centrifuging was kept for IC analysis. The remaining hydrated spheres were weighed using a microbalance and $1 \mathrm{ml}$ Ringers solution was added.

\subsubsection{Sheep uterine arterial embolisation model}

2.2.5.1 Species The five animals used in this study were non-pregnant female sheep aged between 2 and 5 years. The breed was Blanche du Massif Central. The sheep each weighed between 40 and $60 \mathrm{~kg}$ at the beginning of the study.

2.2.5.2 Ethics The study was conducted at Biomatech SAS, France and was approved by Biomatech Ethical Committee prior to implementation. The animals were kept in conditions which conformed to the requirements of farm animals under ECC directive 86/906.

2.2.5.3 Procedure In order to obtain an arterial size in sheep similar to that of a woman with uterine fibroids, hormonal control was required [23]. The menstrual cycle of the sheep was controlled by inserting a sponge impregnated with $40 \mathrm{mg}$ Chronolone (Chrono-gest ${ }^{\circledR}$ Esponge; Intervet, France) inside the vagina of the animal. After 13 days the sponge was removed, and an intramuscular injection of $500 \mathrm{IU}$ of serum derived gonadotropin (Chrono-gest ${ }^{\circledR}$ PMSG; Intervet, France) was administered to achieve ovulation. Pelvic angiography and embolisation of the uterine arteries was then performed within $24 \mathrm{~h}$ of the injection. The animals were fasted $24 \mathrm{~h}$ prior to the procedure.

Embolisation beads $(1 \mathrm{ml})$ were supplied sterile in vials and were mixed with contrast agent prior to embolisation. The beads were suspended at a dilution of 1/10 in a 50:50 mixture of contrast agent and saline in a final volume of $10 \mathrm{ml}$ and gently shaken to homogenize the suspension. This was then split into two $5 \mathrm{ml}$ syringes, one for each feeding artery. A total of $0.5 \mathrm{ml}$ of beads in a $5 \mathrm{ml}$ suspension was used for the embolisation of each uterine artery (this is equivalent to approximately 5000 beads). This volume was selected in order to induce minimal necrosis of the organ and hence allow a proper assessment of the inflammatory response to the beads. The injection 
Table 1 Table showing the number of uterine feeding arteries $(n)$ used in each test group and the corresponding beads that were injected

\begin{tabular}{llll}
\hline Bead type & \multicolumn{2}{l}{ Duration of live exposure to alginate gel bead } \\
\cline { 2 - 4 } & 1 week & 4 weeks & 12 weeks \\
\hline High G & $n=1$ & $n=2$ & $n=2$ \\
High M & $n=1$ & $n=2$ & $n=2$ \\
\hline
\end{tabular}

was performed slowly under fluoroscopic control. After injection of embolic agent, the catheter was purged with approximately $3 \mathrm{ml}$ of saline. The surgery was performed under angiography (Philips BV212). The study did not fully embolise the uterus as this may have impeded assessment of the other factors or caused premature death, by inducing too much tissue necrosis.

One feeding artery was used per test article as shown in Table 1. Animals were killed at 1 week $(n=1,1$ feeding artery per test article), 4 weeks $(n=2,2$ feeding arteries per test article) or 12 weeks ( $n=2,2$ feeding arteries per test article) after embolisation by lethal injection of barbiturate (Dolethal ${ }^{\circledR}$, VETOQUINOL, France).

The uterus, ovaries and local lymph nodes were sampled to allow for the gross and histological examination of the cervix, body and horn.

2.2.5.4 Deliverability (in vivo) Assessments were made at the time of the embolisation procedure: ease of application and handling, reflux or adverse effects at the time of embolisation, duration of embolisation and bead volume injected.

\subsubsection{In vivo analysis procedures}

2.2.6.1 Histological analysis For each level (of the cervix, body and horn segment) one slice was fixed in $10 \%$ buffered formalin and the second slice was fixed in Carnoys fixative. Carnoys fixative consists of $60 \%$ ethanol, $30 \%$ chloroform and $10 \%$ gluteraldehyde and was used because formalin has been reported to dissolve alginate gels [24]. Formalin was still also used as higher sample resolution can be obtained. The tissues were then processed by means of paraffin embedding. Safranin-hematoxylineosin staining was performed. Where beads were found in the histological sections the samples were analysed for signs of inflammation and necrosis.

2.2.6.2 Degradation and distribution Using the histological slides, the beads were noted for signs of degradation in relation to the length of time that they had been in the animals. Possible signs of degradation or resorption were determined as including (i) the presence of cellular infiltration in the bead surface, (ii) the presence of alginate material within the cytoplasm of giant cells and macrophages, (iii) the surface roughness of the bead.

\section{Results and discussion}

\subsection{Compressibility}

Figure 1 shows the comparative moduli $(\mathrm{kPa})$ required to compress various beads. The trend observed is the same as that seen by other compression tests in the literature [25]. There is no statistical significance $(P>0.05$; Bonferonni corrected Student's $t$-test) between High M and High G alginate beads. Alginate beads are most similar in compressibility to Bead Block (Bonferroni corrected Student's $t$-test, $P>0.05(\mathrm{G}) ; P>0.05(\mathrm{M}))$.

\subsection{Deliverability}

The compressibility analysis of the alginate beads indicated that the delivery should be similar to beads already on the market. However whilst the compressibility was similar, the compressibility method does not establish the robustness, fragmentation potential or elastic recovery speed of the beads. In order to establish these properties, it was necessary to test the beads in a clinically relevant scenario. This was achieved by forcing the beads through a $2.7 \mathrm{Fr}$ Progreat microcatheter that is commonly used in embolisation procedures. Images of the alginate beads post delivery can be seen in Fig. 2.

The in vitro delivery of the alginate beads in omnipaque contrast media, offered some resistance with an average score of 2.5 when being delivered through the catheter. When beads had already been delivered in the catheter lumen and the catheter was not flushed with saline, slightly higher resistance was felt during the next bead injection; with a delivery score of 2 . No fragmentation was noted for both high $\mathrm{M}$ and high $\mathrm{G}$ beads.

The delivery of the beads in the sheep uterine artery embolisation model was quite similar for the two arteries to be embolised respectively; $9.8 \mathrm{~s} \pm 3.0$ (SD) for the left and $10.2 \mathrm{~s} \pm 3.9$ (SD) for the right artery showing consistency. The mean score calculation for those parameters per type of bead did not show marked difference (Table 2). All radiologists graded both bead types as good with regards to their ease of handling and application This was consistent with the in vitro assessment. In addition the embolisation times were very similar for all injections $(\approx 10 \mathrm{~s})$; another demonstration of facile delivery. Further analysis of the data showed that any variation in the duration of injection was attributable to the surgeon that performed the injection on the day rather than to the beads themselves (Student's $t$ test, $P<0.05)$. Moreover, regardless of the interventional 
Fig. 1 Modulus of M and $\mathrm{G}$ alginate beads alongside commercially-available embolic beads $(n=5 ; \pm \mathrm{SD})$. Alginate beads are most similar in compressibility to Bead Block (Bonferroni corrected Student's $t$-test, $P>0.05(\mathrm{G}) ; P>0.05$ (M))

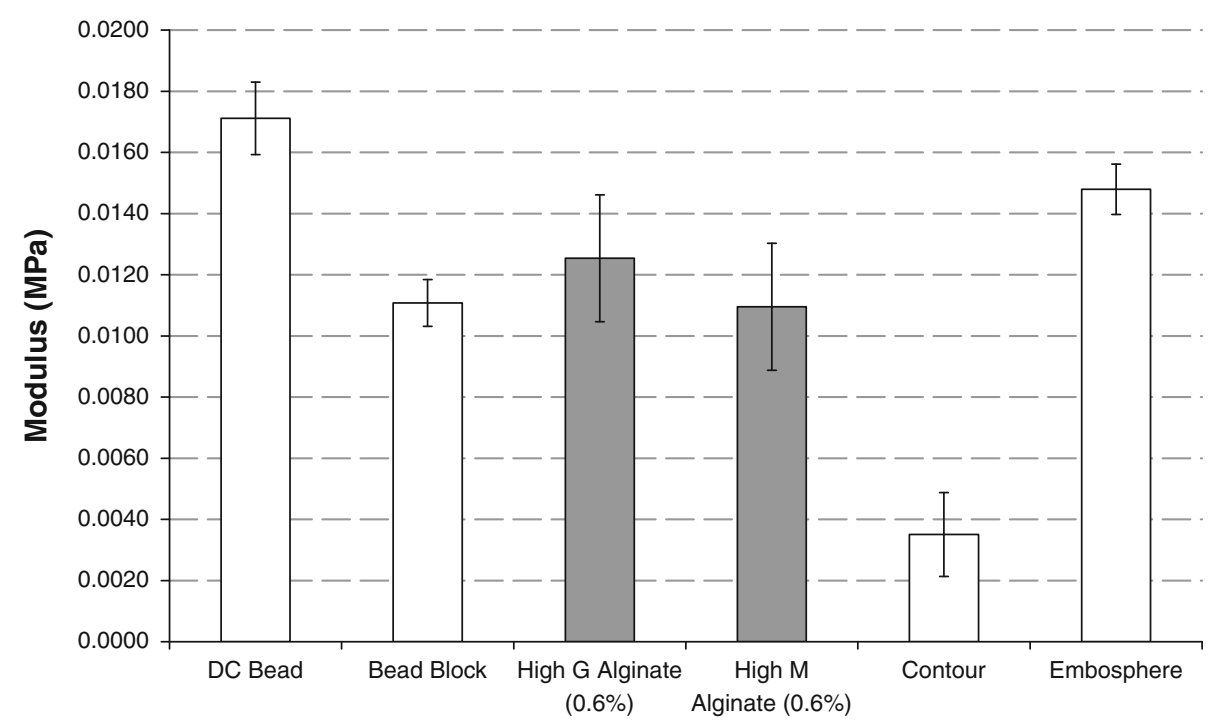

Fig. 2 Phase contrast image of high $\mathrm{G}$ (left) and high M (right) beads post catheter delivery. No fragmentation was observed
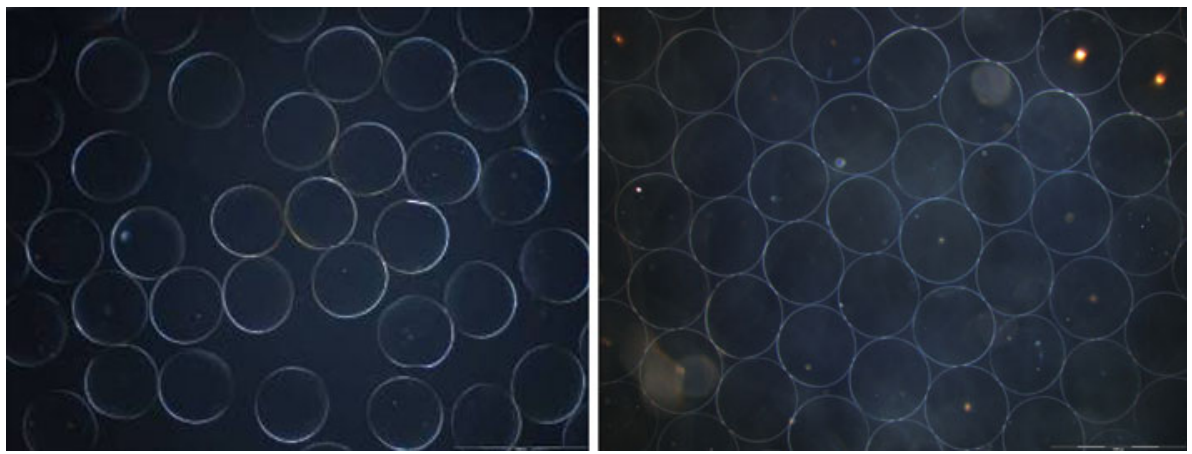

Table 2 Occlusion times and potential to restore the blood vessel of embolic materials used in transcatheter embolisation [23]

\begin{tabular}{llll}
\hline Material & Vessel size & Duration of occlusion & $\begin{array}{l}\text { Potential for restoring } \\
\text { vascular continuity }\end{array}$ \\
\hline Autologous blood clot & Branch artery & $8-24 \mathrm{~h}$ & Excellent \\
Gelfoam particles & Arterioles & $2-3$ weeks & Good \\
Gelfoam powder & Capillary bed & $\geq 3$ weeks & Poor \\
Ivalon (PVA, dehydrated particles) & Small Arteries & Semi-permanent & Limited \\
\hline
\end{tabular}

radiologist used, had there been a blockage the time taken to inject the beads would have increased noticeably compared to the average for that user. No catheter blockages occurred during any of the procedures.

\subsection{Bead degradation}

\subsubsection{In vitro: size, shape and weight change}

The weight change of the centrifuged hydrated beads (Fig. 3a) followed a similar profile to as the size difference (Fig. 3b). Both showed an increase during the first week in Ringers solution and then began to decrease after 4 weeks.
High $M$ beads showed greater size and weight increase than high $\mathrm{G}$.

\subsubsection{In vivo: gross examination}

At first week a slight reddening of the uterus was observed and attributed to embolisation induced necrosis. Clear macroscopic signs of necrosis or inflammatory reaction could not be observed in the gross specimens for the 4 and 12 week time period. The lack of any reddening at 4 and 12 weeks as attributed to the embolised areas finding new blood supplies as a result of the small bead volumes used. 

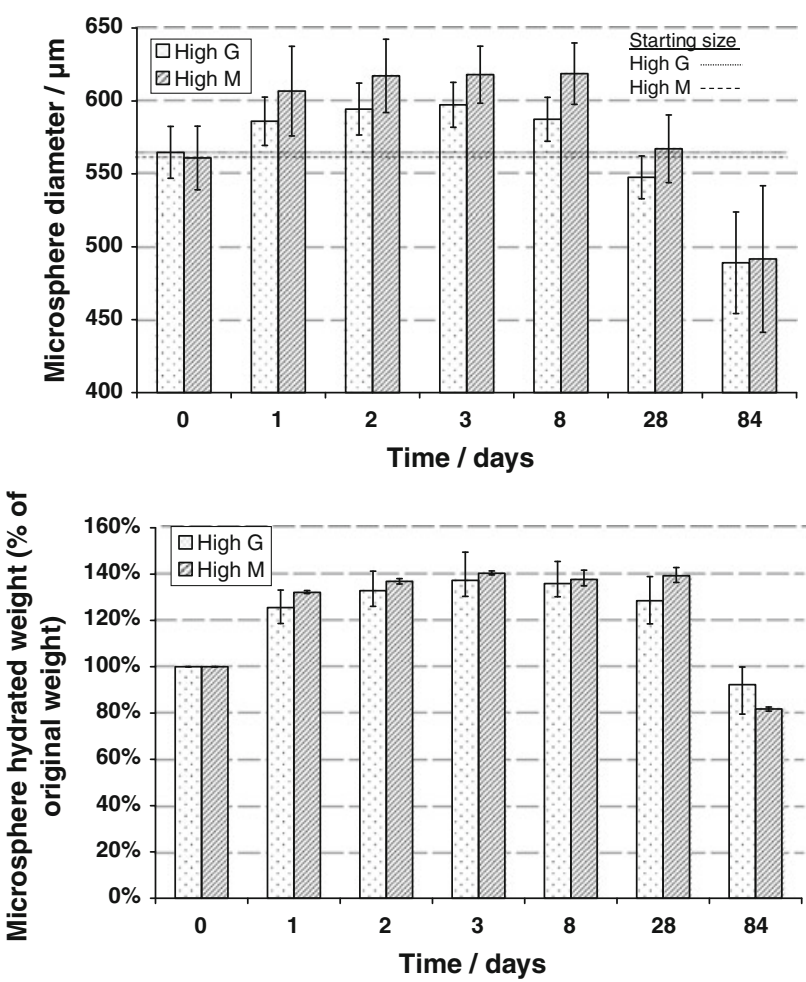

Fig. 3 Change in $M$ and $G$ alginate gel beads a diameter (mean, $\pm \mathrm{SD},(n=100)$ ) and $\mathbf{b}$ hydrated weight (\% original weight, $\pm \max /$ min, $(n=3))$, in $1 \mathrm{ml}$ Ringer's solution at $37^{\circ} \mathrm{C}$ for 12 weeks. The Ringer's solution was removed and replaced at each time point. Initially, the size increased over the first few days, but decreased back to the original size after 4 weeks and then dropped below the original size after 12 weeks

\subsubsection{In vivo: histopathological analysis}

Generally, beads were detected in one of four environments. The bead was found either completely occluding an artery or within an artery surrounded by white space. The white space is likely to be due to the bead dehydrating during the processing step [26]. The third environment where the presence of beads was detected was the observation of a circular space left behind within an artery. The presence of a bead was shown by the compression of the intima and media arterial layers; non-embolised arteries of a similar size have thicker walls and non-circular elastic lumen. In some instances the presence of a bead was apparent through the observation of a circular gap in the thrombus within an artery. The beads were sometimes observed in a combination of these situations and were not always observed as single beads. On occasion a longitudinal cut of an artery would reveal a line of beads or a cluster of beads could be detected in a larger artery.

The alginate beads within the arterial lumen are not necessarily spherical because the slice cannot always be taken perpendicular to the artery. Beads may seem elongated or misshapen if the section had been taken at a non- perpendicular angle. In addition odd shapes may be observed if the slice was taken at an arterial junction. Also, the processing of the tissue and thin histology sections mean that the likelihood of observing a bead is small and consequently only few beads were observed in all of the sections. It is worth noting that the lack of any beads does not necessarily mean that any were lost through degradation.

The extent of necrosis and inflammation observed for the samples was assessed and scored on a simple threegrade scale (grade $0=$ absent, grade $1=$ moderate $(<50 \%$ of the observed area), grade $2=$ extensive $(>50 \%$ of the observed area). At first week, the mean necrosis score was only $0.46 \pm 0.71$, indicating less than moderate necrosis; this is to be expected given the small amount of embolic used was regulated to limit the degree of necrosis occurring. Necrosis was indicated by light-pink staining under $\mathrm{H} \& \mathrm{E}$ of a largely acellular nature with lack of visible nuclei, mostly associated with sections containing multiple beads where the embolic effect would be more severe (Fig. 4, black arrows). There was no evidence of necrosis at either the 4 week or 12 week time points, as the tissue has remodelled. Inflammation was similarly only very mild (average score over all samples $0.25 \pm 0.43$ ) and again, only observed at the 1 week time period.

At all time points alginate beads were observed within arteries post mortem. Cellular infiltration and surface roughness were infrequent but were observed at all time points, for both bead types (Fig. 5). This demonstrated that this method of degradation was not time nor bead specific. Generally, if cellular infiltration did occur, the site was often located near signs of thrombus, which is likely to have been induced by embolisation. In addition, at all time points, round smooth beads were found in the lumen of arteries without signs of thrombus, inflammatory cells or degradation (Fig. 5). This suggests that the beads were not

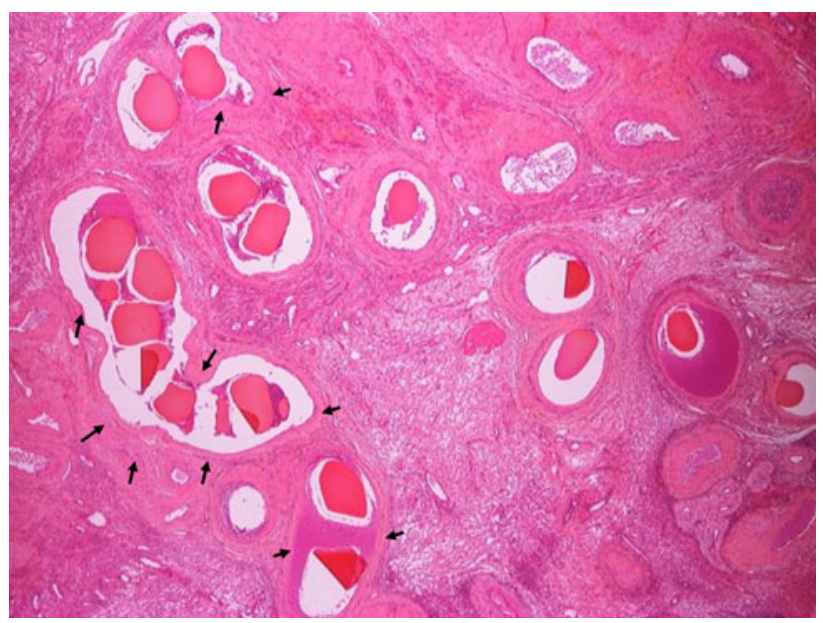

Fig. 4 Example of multiple beads (high $\mathrm{G}$ alginate) at 1 week, with some areas of associated necrosis (arrows) surrounding the vessels 
Fig. 5 Images of high $\mathrm{G}$ and high $\mathrm{M}$ alginate beads after 1,4 and 12 weeks in an embolisation environment. Examples of smooth and roughedged beads can be observed for both types at all time points

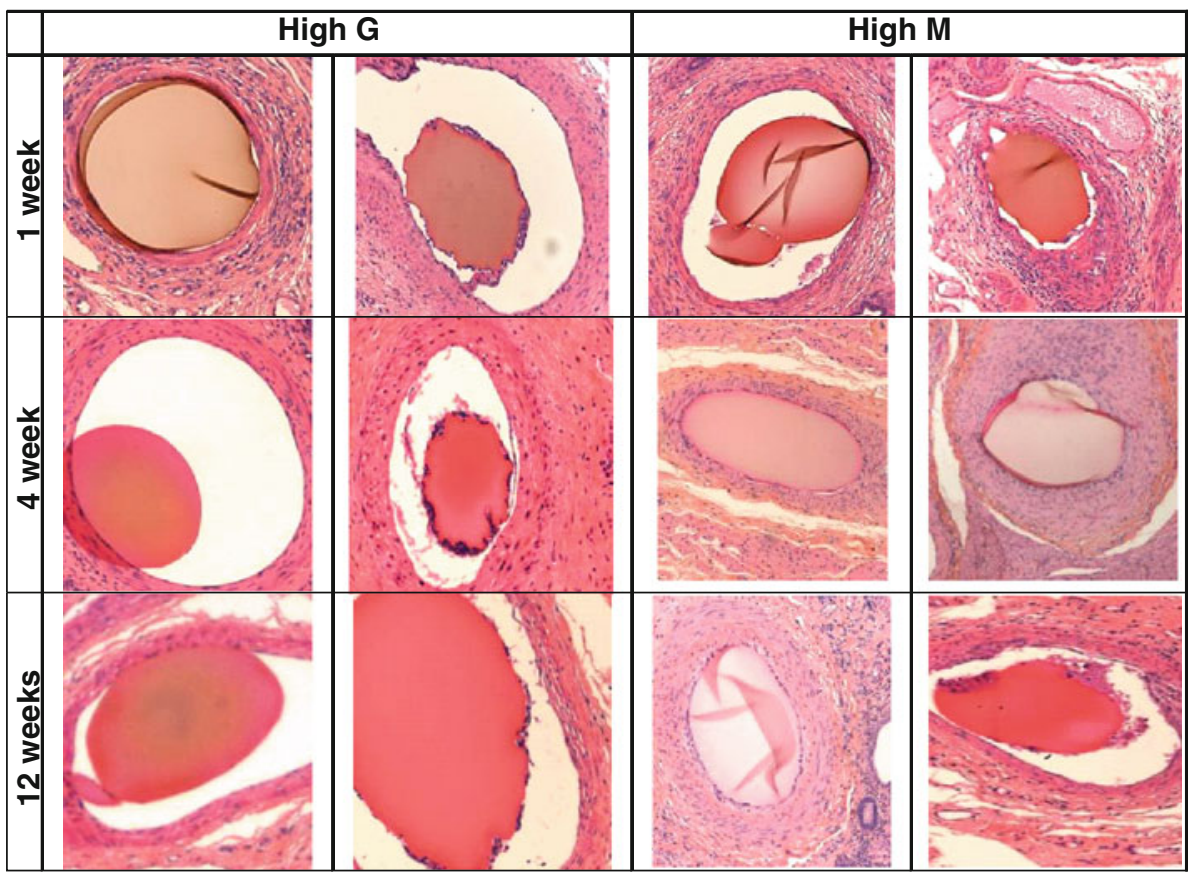

degrading simply due to their presence within the arterial system. Some evidence of further degradation by extensive cellular infiltration was observed at some, but not all, time points. Some examples are given in (Fig. 6). None of the alginate beads however, were fully degraded within 12 weeks.

The level of bead degradation 1, 4 and 12 weeks postimplantation in vivo did not follow any trends with respect to time (Fig. 5). In addition, degradation could not be monitored by size as the sectioning was effectively random so beads could not be shown to have been sliced at the largest diameter, thereby making any comparison void. The sample number was too low to overcome this.

\section{Discussion}

In embolic procedures the ease of delivery of the embolic is imperative, unwanted catheter blockage adds time and pressure to the process; limiting the control the interventional radiologist has. The high purity, high molecular weight alginate gel beads (both high $\mathrm{G}$ and high $\mathrm{M}$ ) showed good deliverability in vitro, and in vivo. The lack of fragmentation will prevent any unwanted premature embolisation due to particulates clumping together. The data obtained from compression testing shows that neither a higher $\mathrm{G}$ or $\mathrm{M}$ content has any significant effect on the compressibility of the alginate gel beads; the in vivo data also showed no obvious difference between the two bead types. The in vitro compressibility of the calcium crosslinked high molecular weight alginate beads was most similar to Bead Block, an embolic bead that is currently used for UFE [25, 27].

The main physical difference between the in vitro setup and the reality in vivo is the extent to which $1 \mathrm{ml}$ represented the sink conditions in an embolisation capacity; this is yet to be fully determined.

In vivo, inflammatory cell infiltration and bead surface roughness appeared to be often thrombus-associated, whereas the beads with smooth surfaces appeared to lack this association. This suggests that whilst an alginate bead can undergo degradation, this may be accelerated by the infiltration of surrounding thrombus by inflammatory cells. Some beads will be exposed to more thrombus than others as a consequence of their relative position within the embolised vessel; those more proximal or distal in the column of occluding beads for instance. This accounts for the interbead variability observed. The reorganisation of tissue and bead degradation makes the system dynamic and in time allows previously unexposed beads to become eventual targets for inflammatory cells. Consequently, both pristine and misshapen beads can be seen at all time points. When compared to studies of a range of other embolic agents on the impact on inflammatory tissue and foreign body reaction in porcine liver [28] or kidney [29] models, our findings here were consistent with a low level inflammatory reaction in the uterine arteries. The excellent overall tolerance of the alginate beads in vivo is an expected consequence of the ultra-high purity alginate used to synthesise the beads. These observations could be important when considering new materials suitable for use in biodegradable embolic devices. 
Fig. 6 Cellular infiltration of a high $M$ and $\mathbf{b}$ high $G$ after 4 weeks in sheep uterine artery
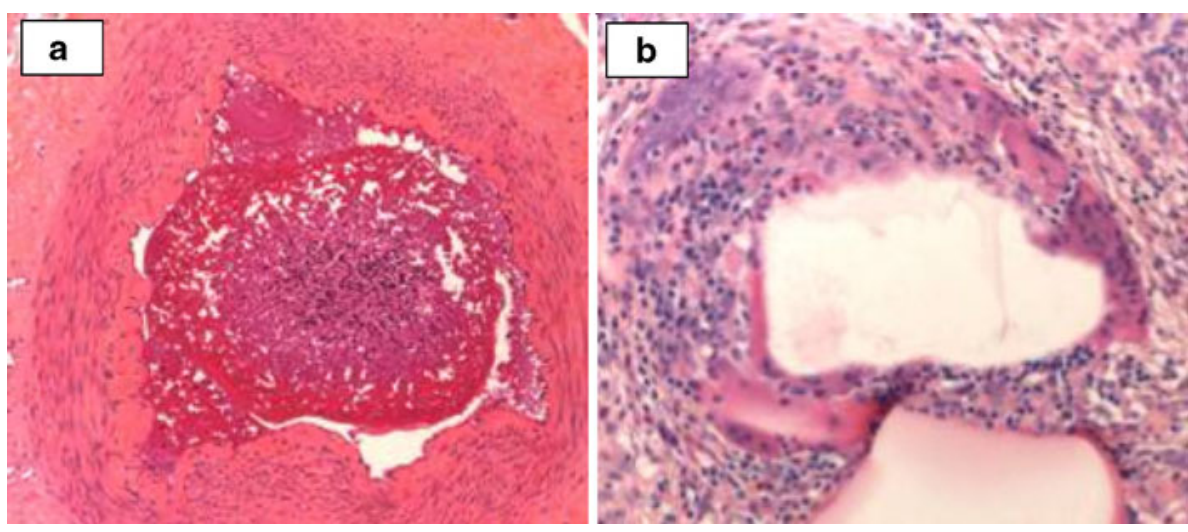

In vitro degradation began to be observed after 12 weeks under pseudo embolisation conditions. Lang [30] composed a table of the occlusion times and potential to restore the blood vessel for embolic materials used in transcatheter embolisation. Although this table illustrates data for the occlusion of arteries of different sizes, it is clear that 12 weeks degradation is long enough to maintain the occlusion, but is probably too late to enable restoration of vascular continuity within that blood vessel. In vivo however, no significant difference was noted between the $\mathrm{G}$ or $\mathrm{M}$ beads with regards to size and neither showed signs of degradation after 1,4 and 12 weeks. The discrepancy may be due to the biological environment to which an embolised microsphere is exposed.

Compared to the 2-3 week degradation time of Gelfoam, the in vitro degradation of 4-12 weeks may mean that these alginate beads may be too permanent to restore vascular connection. However, patients commonly express worries about foreign materials remaining in the body in uterine fibroid embolisation [31] and whilst this may or may not be a physiological problem, reduction of additional angst for the patient is desirable. An embolic, made from what could be considered a natural material, which can perform its function and then be degraded and excreted from the body over time, could be attractive to the patient. The alginate beads tested here could offer this potential. The study presented here however, aimed to evaluate tissue reaction to the alginate material and its degradation potential. Although the mechanical properties of the alginate beads would suggest that they have equivalent deformation properties to other commercially available embolisation microspheres, further work is required in an alternative relevant model to understand the effectiveness of the embolisation procedure and the tendency for recanalisation of the vessel.

\section{Conclusions}

High molecular weight alginate gel beads have been evaluated in vitro and in vivo to establish whether their physical properties show the capability to be considered as an embolisation device. Both the in vitro and in vivo data demonstrate good delivery, with no tendency to block catheters or fragment owing to compressibility properties comparable to current commercially-available embolic beads. In vitro, swelling was noted almost immediately and after 12 weeks the first signs of degradation were observed, although little difference was noted in vivo. This study has shown that high molecular weight alginate gel beads are well tolerated by the body, but beads susceptible to inflammatory cell infiltration were more often associated with surrounding thrombus. The thrombus presumably acts as a focus for inflammatory cell infiltration during remodelling of the vessel post embolisation. The beads were shown to be easy to handle and were still observable largely intact in histological sections after 3 months in vivo. The beads handle well and are robust enough to be delivered through a 2.7 Fr microcatheter, which is a commonly used product in UFE procedures. This study has demonstrated that high molecular weight, high purity alginate beads can be considered as semi-permanent embolisation beads, with the potential to resorb over time.

Acknowledgements RF would like to acknowledge funding for a $\mathrm{PhD}$ provided under a Fellowship of the Royal Commission of 1851. We would like to thank Dr Michel Wassef, Service d'Anatomie Pathologique, Hôpital Lariboisière for his kind opinion on some of the histological analyses. This study was in part funded by the EU under Project Number FP6 512956, COOP-CT-2004-513121-POSBEADD.

Open Access This article is distributed under the terms of the Creative Commons Attribution Noncommercial License which permits any noncommercial use, distribution, and reproduction in any medium, provided the original author(s) and source are credited.

\section{References}

1. Jen AC, Wake MC, Mikos AG. Review: hydrogels for cell immobilization. Biotechnol Bioeng. 1996;50(4):357-64.

2. Orive $\mathrm{G}$, et al. Biocompatibility of microcapsules for cell immobilization elaborated with different type of alginates. Biomaterials. 2002;23(18):3825-31. 
3. Fischer FG, Dorfel H. Polyuronic acids in brown algae. Hoppe Seylers Z Physiol Chem. 1955;302(4-6):186-203.

4. Smidsrød O, Draget KI. Chemistry and physical properties of alginates. Carbohydr Eur. 1996;14:6-12.

5. Draget KI, Skjak-Braek G, Smidsrod O. Alginate based new materials. Int J Biol Macromol. 1997;21(1-2):47-55.

6. Haug A, Smidsrod O. The effect of divalent metals on the properties of alginate solutions. II. Comparison of different metal ions. Acta Chem Scand. 1965;19:341-51.

7. Haug A, Larsen B, Smidsrød O. The degradation of alginates at different pH values. Acta Chem Scand. 1963;17:1466-8.

8. Alsberg E, et al. Regulating bone formation via controlled scaffold degradation. J Dent Res. 2003;82(11):903-8.

9. Kikuchi A, et al. Effect of $\mathrm{Ca}^{2+}$-alginate gel dissolution on release of dextran with different molecular weights. J Control Release. 1999;58(1):21-8.

10. Golzarian J, Sun S, Sharafuddin MJ. Vascular embolotherapy: a comprehensive approach. Medical radiology, 0942-5373. Diagnostic imaging. 2006, Berlin; [London]: Springer. 2 v.

11. Ravina JH, et al. Arterial embolisation to treat uterine myomata. Lancet. 1995;346(8976):671-2.

12. ACOG Committee Opinion. Uterine artery embolization. Obstet Gynecol. 2004;103(2):403-4.

13. Worthington-Kirsch R, et al. The Fibroid Registry for outcomes data (FIBROID) for uterine embolization: short-term outcomes. Obstet Gynecol. 2005;106(1):52-9.

14. Edwards RD, et al. Uterine-artery embolization versus surgery for symptomatic uterine fibroids. N Engl J Med. 2007;356(4):360 70.

15. Mara M, et al. Midterm clinical and first reproductive results of a randomized controlled trial comparing uterine fibroid embolization and myomectomy. Cardiovasc Interv Radiol. 2008;31(1):73-85.

16. Pinto I, et al. Uterine fibroids: uterine artery embolization versus abdominal hysterectomy for treatment - a prospective, randomized, and controlled clinical trial. Radiology. 2003;226(2):42531.

17. Volkers NA, et al. Uterine artery embolization versus hysterectomy in the treatment of symptomatic uterine fibroids: 2 years' outcome from the randomized EMMY trial. Am J Obstet Gynecol. 2007;196(6):519 e1-11.

18. Zimmermann U, Behringer M. Verfahren Zur Gewinnung Hochgereinigter Alginate. In: EP1109837. 1999, CellMed AG.
19. Heile AM, et al. Cerebral transplantation of encapsulated mesenchymal stem cells improves cellular pathology after experimental traumatic brain injury. Neurosci Lett. 2009;463(3):17681.

20. Farrell K, et al. GLP-1 CellBeads enhance myocardial angiogenesis and improve LV function. Eur Heart J. 2009;30(Abstract Supplement): 495 .

21. Wolters GHJ, et al. A versatile alginate droplet generator applicable for microencapsulation of pancreatic islets. J Appl Biomater. 1992;3(4):281-6.

22. Leo WJ, McLoughlin AJ, Malone DM. Effects of sterilization treatments on some properties of alginate solutions and gels. Biotechnol Prog. 1990;6(1):51-3.

23. Pelage JP, et al. Uterine artery embolization in sheep: comparison of acute effects with polyvinyl alcohol particles and calibrated microspheres. Radiology. 2002;224(2):436-45.

24. Becker TA, Kipke DR, Brandon T. Calcium alginate gel: a biocompatible and mechanically stable polymer for endovascular embolization. J Biomed Mater Res. 2001;54(1):76-86.

25. Lewis AL, et al. Comparative in vitro evaluation of microspherical embolisation agents. J Mater Sci Mater Med. 2006;17(12):1193-204.

26. Laurent A, et al. Location of vessel occlusion of calibrated trisacryl gelatin microspheres for tumor and arteriovenous malformation embolization. J Vasc Interv Radiol. 2004;15(5):491-6.

27. Kroencke TJ, et al. Acrylamido polyvinyl alcohol microspheres for uterine artery embolization: 12-month clinical and MR imaging results. J Vasc Interv Radiol. 2008;19(1):47-57.

28. Stampfl U, et al. Experimental liver embolization with four different spherical embolic materials: impact on inflammatory tissue and foreign body reaction. Cardiovasc Interv Radiol. 2009;32(2):303-12.

29. Stampfl S, et al. Immunohistochemical characterization of specific inflammatory tissue reactions following embolization with four different spherical agents in the minipig kidney model. J Vasc Interv Radiol. 2009;20(7):936-45.

30. Lang EK. Transcatheter embolization of pelvic vessels for control of intractable hemorrhage. Radiology. 1981;140(2):331-9.

31. Reynolds A. Diagnosis and management of uterine fibroids. Radiol Technol. 2007;79(2):157-78; quiz 179-82. 